\title{
The circulating microRNA-200 family in whole blood are potential biomarkers for high-grade serous epithelial ovarian cancer
}

\author{
ADAM PENDLEBURY ${ }^{1}$, NATALIE J. HANNAN ${ }^{2}$, NATALIE BINDER ${ }^{2}$, SALLY BEARD $^{2}$, \\ MONICA MCGAURAN ${ }^{2}$, PETER GRANT ${ }^{1}$, STEPHEN TONG ${ }^{2}$ and CLARE L. WHITEHEAD ${ }^{2}$ \\ Departments of ${ }^{1}$ Gynaecological Oncology and ${ }^{2}$ Obstetrics and Gynaecology, \\ University of Melbourne, Mercy Hospital for Women, Heidelberg, Vic 3084, Australia
}

Received September 21, 2016; Accepted December 30, 2016

DOI: 10.3892/br.2017.847

\begin{abstract}
Epithelial ovarian cancer (EOC) is the leading cause of mortality with regard to gynaecological cancer. There is no effective biomarker and therefore prognosis is poor as the majority of cases are not diagnosed until advanced disease is present. MicroRNAs (miRs) are dysregulated in ovarian cancer tissue and are present in the circulation. The aim of the present study was to investigate whether circulating miRs from the miR-200 family served as potential candidate biomarkers for the early detection of EOC. Whole blood and ovarian tissue were collected from women with early (stage I/II, n=4), advanced EOC (stage III/IV, n=4), and women with benign ovarian masses $(n=5)$. A panel of 5 miRs were studied in whole blood and ovarian tissue using Taqman RT-PCR miR assays. The expression of circulating miR-200a, miR-200b and miR-200c were upregulated $(\mathrm{P}<0.05)$ in ovarian cancer compared to controls, correlated with the stage of disease and reflected tissue expression. Despite a trend, there was no significant increase in the expression of miR-21 and miR-210 in the present study. In conclusion, the circulating miR-200 family may be promising candidate biomarkers for EOC that require validation in a larger study.
\end{abstract}

\section{Introduction}

Ovarian cancer is the fifth most lethal cancer affecting women, and accounts for the majority of mortalitites from gynaecological cancer (1). Epithelial ovarian cancer (EOC) accounts for $90 \%$ of all cases of ovarian cancer and is typically a 'silent killer' because patients do not present with symptoms until advanced disease is present. As a result, $70 \%$ of patients

Correspondence to: Dr Clare L. Whitehead, Department of Obstetrics and Gynaecology, University of Melbourne, Mercy Hospital for Women, 163 Studley Road, Heidelberg, Vic 3084, Australia

E-mail: clarew@unimelb.edu.au

Key words: epithelial ovarian cancer, biomarker, microRNA have stage III/IV (International Federation of Gynaecology and Obstetrics-FIGO staging) advanced EOC at the time of diagnosis, resulting in a 5-year survival $<30 \%$ (2). By contrast, if EOC is diagnosed early, at stage I, the 5-year survival rate is $90 \%$. This high survival rate with early disease diagnosis highlights the need for an accurate test to detect ovarian cancer. Screening with CA 125 and/or pelvic ultrasound has been extensively studied but has not been shown to improve mortality, due to poor sensitivity and specificity for ovarian cancer (3). Novel biomarkers are therefore required to enhance screening strategies and improve survival rates.

MicroRNAs (miRs) are small non-coding RNAs that are essential for regulating gene expression. They play important roles in the regulation of a wide variety of genes by binding to the 3'-untranslated regions of the target mRNA and are involved in the pathogenesis of various types of cancer (4). The fact that miRs circulate in the blood where they may reflect gene expression in a distant tumour, has led to enthusiasm for the role of circulating miRs as biomarkers for cancer detection and prognosis. Multiple miRs appear to be dysregulated in EOC tissue but it is unclear whether circulating miRs can be used to identify women with EOC compared to controls.

We selected a panel of five miRs that have been shown to be dysregulated in ovarian cancer tissue. The miR-200 family (miR-200a, miR-200b and miR-200c) plays a key role in initiating cancer development and invasion by regulating the transformation of epithelial to mesenchymal cells (5). Hypoxia-regulated miRs are also involved in cancer progression, modulating the cell response to increasing hypoxia as the tumour outgrows its vascular supply (6). Both miR-21 and miR-210 are consistently upregulated in response to hypoxia and therefore may be upregulated in EOC compared to controls. In the present study, we examined the expression of circulating miRs in blood and tissue in EOC and benign ovarian masses to determine whether they are potential biomarkers for EOC.

\section{Materials and methods}

Study subjects. All the subjects were recruited from the Mercy Hospital for Women, a tertiary referral hospital in Australia in 2013-2014. Written informed consent was obtained prior to 
inclusion and the present study was approved by the Mercy Health Human Research and Ethics Committee, Melbourne, Australia no. R11/34.

Whole blood was collected prior to surgery in women suspected of EOC. Biopsies of ovarian tissue were collected intraoperatively and processed immediately. Cases $(n=8)$ were confirmed after a multidisciplinary meeting and full histopathological diagnosis of type, grade and FIGO stage of ovarian cancer was confirmed. Controls $(n=5)$ were aged-matched but found to have benign ovarian masses surgically and histopathologically. Patients with a history of previous or recurrent cancer, as well as non-EOC cases were excluded.

Sample collection. Peripheral whole blood samples $(2.5 \mathrm{ml})$ were collected in PAXgene blood RNA tubes (PreAnalytix, Hombrechtikon, Switzerland). As per the manufacturer's instructions, the samples were stored at room temperature for 24-72 h, transferred to $-20^{\circ} \mathrm{C}$ for $24 \mathrm{~h}$, and then stored at $-80^{\circ} \mathrm{C}$ until processing.

Ovarian tissue biopsies were collected intraoperatively and immediately transferred into RNALater (Qiagen, Hilden, Germany) and snap frozen in liquid nitrogen. They were then stored at $-80^{\circ} \mathrm{C}$ until processing.

miRNA preparation. miRNA was extracted from peripheral whole blood using the PAXgene Blood miRNA kit (PreAnalytix) and automated QiaCube (Qiagen) protocol according to the manufacturer's instructions. Genomic DNA was removed using DNase treatment, and total RNA was eluted and stored at $-80^{\circ} \mathrm{C}$ if not used immediately. The concentration of total RNA was quantified by NanoDrop 1000 (Nanodrop Technologies; Thermo Fisher Scientific, Waltham, MA, USA).

miRNA was extracted from ovarian tissue using the RNeasy Kit (Qiagen). In brief, ovarian tissue was homogenized in buffer. The lysate was centrifuged at 8,000 $\mathrm{x}$ g for $3 \mathrm{~min}$, and the supernatant added to $350 \mu \mathrm{l}$ of $70 \%$ ethanol. The sample was then run through the RNeasy spin column and centrifuged with buffers as per protocol. The final RNA was eluted into $30 \mu \mathrm{l}$ of RNase free water and stored at $-80^{\circ} \mathrm{C}$, if not used immediately.

RT-PCR. Specific miRNA Taqman assays (Applied Biosystems Life Technologies, Foster City, CA, USA) were used for the quantification of the miRNAs according to the manufacturer's instructions. To assess miR-200a, miR-200b and miR-200c the High-Capacity cDNA Reverse Transcription Kit was used. Total RNA (150 ng) was reverse transcribed for each target miRNA and the housekeeping miRNA miR-191 was used for normalization. To assess miR-21 and miR-210 the TaqMan MicroRNA Reverse Transcription kit was used (with a cDNA conversion and preamplification step) for the two miRs (miR-21 and miR-210) and the housekeeping miRNA RNU-48. Following cDNA generation qPCR was performed in duplicate, with multiple negative controls, using TaqMan Universal PCR Master Mix II on a CFX 384 (Bio-Rad, Berkeley, CA, USA) with the following cycling conditions: $95^{\circ} \mathrm{C}$ for $10 \mathrm{~min}$, and 40 cycles of $95^{\circ} \mathrm{C}$ for $15 \mathrm{sec}$, and $60^{\circ} \mathrm{C}$ for $1 \mathrm{~min}$. Fold-changes in expression were determined by the
Table I. Clinical characteristics of EOC cases and benign controls.

\begin{tabular}{lll}
\hline Characteristics & Case & Control \\
\hline Age (years) & 56.3 & 63.5 \\
Benign cystadenoma (n, \%) & 0 & $5(100)$ \\
FIGO stage (n, \%) & & 0 \\
I-II & $4(50)$ & \\
III-IV & $4(50)$ & 0 \\
Grade (n, \%) & & \\
Low & 0 & \\
High & $8(100)$ & \\
\hline
\end{tabular}

EOC, epithelial ovarian cancer.

comparative CT method normalized against the mean expression of the housekeeping gene.

Statistical analysis. Data were statistically analyzed using R version 2.12.0 (http://www.r-project.org) or GraphPad Prism v 5 (GraphPad Software, Inc., La Jolla, CA, USA). Differences in RT-PCR gene expression were assessed using the Mann-Whitney U or Kruskal-Wallis test, or where the data were considered normally distributed, the t-test or ANOVA. Patient characteristics were compared using $\chi^{2}$ where appropriate. Data were presented as mean \pm standard deviation. $\mathrm{P}<0.05$ was considered to indicate a statistically significant difference.

\section{Results}

Clinical characteristics. Clinical characteristics for the EOC cases and controls are presented in Table I. The EOC patients had predominately ( $>80 \%$ ) high-grade serous carcinoma of the ovary, $50 \%$ of whom had stage I/II and $50 \%$ had stage III/IV at the time of diagnosis.

Circulating miRNA expression in EOC. We examined the expression of miRs in the circulation to determine their potential to be blood-based biomarkers for EOC (Fig. 1). We found an increased expression of miR-200a, miR-200b and miR-200c in the blood in cases with EOC compared to controls and the expression increased as the stage of EOC increased. The circulating expression of miR-200a in stage I/II EOC was 5-fold greater than the controls and 8 -fold greater for stage III/IV $(\mathrm{P}<0.05)$. The circulating expression of miR-200b in stage I/II EOC was 2.5-fold greater than controls and 5.6-fold greater for stage II/IV $(\mathrm{P}<0.05)$. The circulating expression of miR-200c in stage I/II EOC was 4.7-fold greater than controls and 23.3-fold greater for stage II/IV $(\mathrm{P}<0.05)$. The expression of circulating miR-21 and miR-210 was increased in EOC but did not meet statistical significance in this small cohort.

miRNA expression in EOC tissue. We also compared the expression of the miRs in ovarian tissue collected intraoperatively from women with benign ovarian masses 

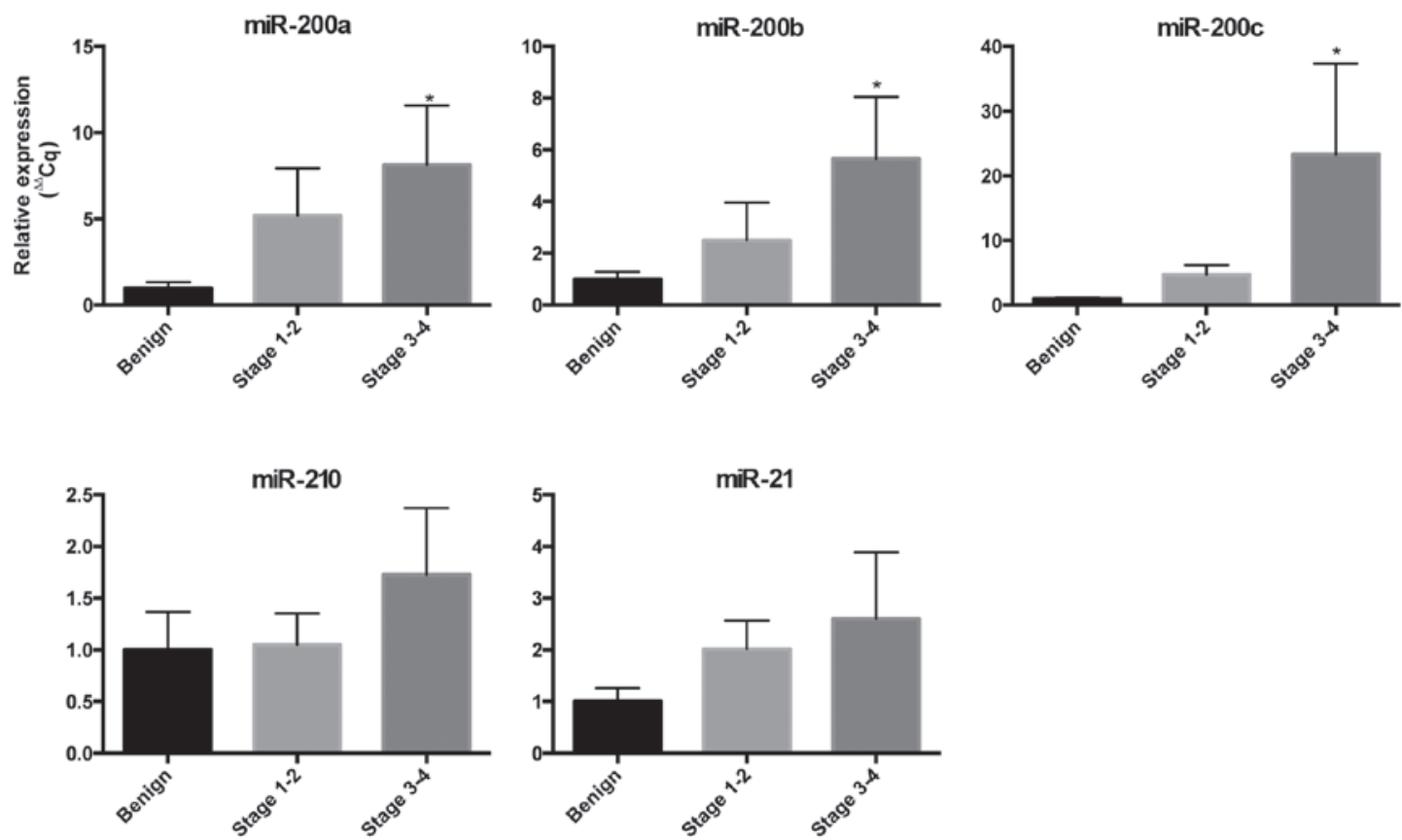

Figure 1. Expression of circulating microRNAs (miRs) in women with epithelial ovarian cancer (EOC). miRNA expression of miR-200a, miR-200b, miR-200c, miR-21 and miR-210 in whole blood collected from women with early EOC (stage I/II, n=4) and advanced EOC (stage III/IV, n=4), compared to controls with benign ovarian masses $(n=5)$. Expression normalized against miR-191 and RNU-48. Data are presented as mean \pm standard error of the mean. Mann-Whitney test was used for statistical comparison to controls.
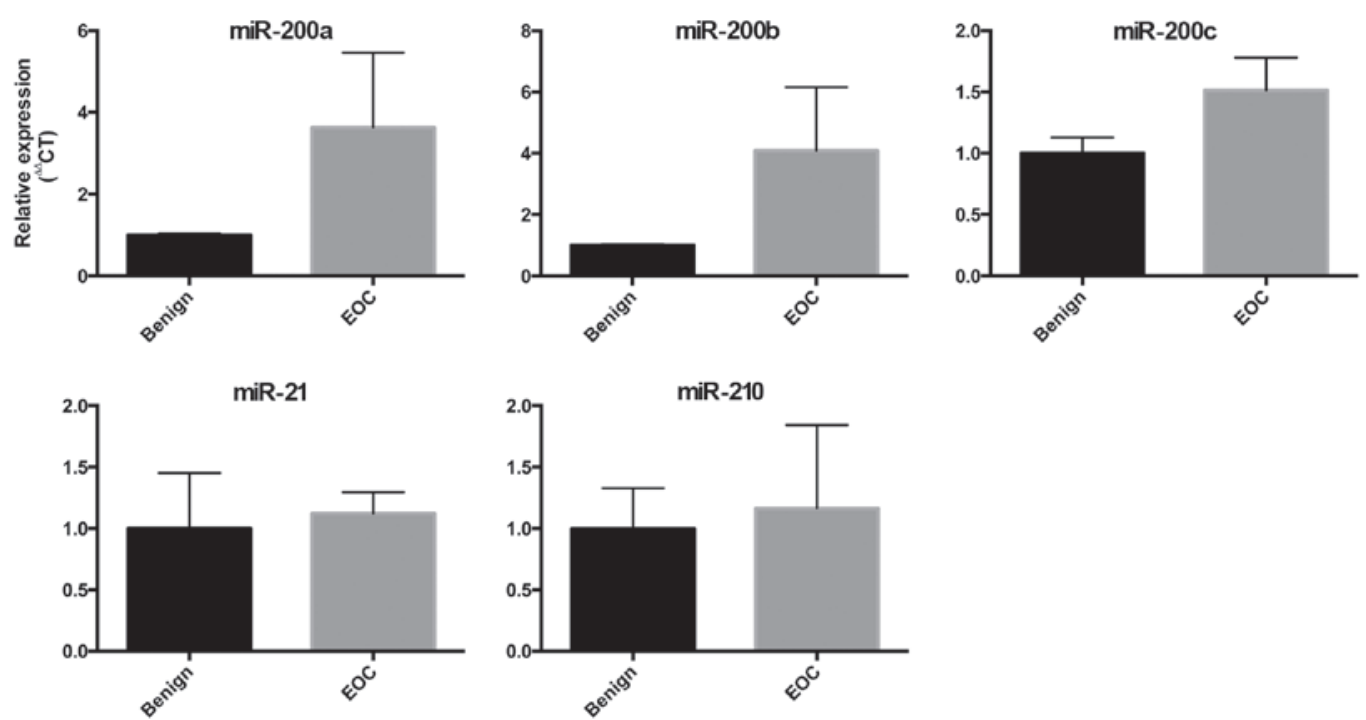

Figure 2. Expression of microRNAs (miRs) in ovarian tissue from women with epithelial ovarian cancer. miRNA expression of miR-200a, miR-200b, miR-200c, miR-21 and miR-210 in ovarian tissue collected intraoperatively from women with EOC $(n=4)$, compared to controls with benign ovarian masses $(\mathrm{n}=6)$. Expression normalized against miR-191 and RNU-48. Data are presented as mean \pm standard error of the mean. Mann-Whitney test was used for statistical comparison to controls. EOC, epithelial ovarian cancer.

and women with EOC. The expression of the miR-200 family showed a trend towards increased expression in ovarian tissue from women with EOC, but this did not meet statistical significance. There were no differences in the expression of miR-210 or miR-21 in this small cohort (Fig. 2).

\section{Discussion}

Early diagnosis of EOC is dependent on the development of a reliable biomarker that can discriminate between benign and malignant pelvic tumours. To the best of our knowledge, currently, no screening modality for EOC exists. miRs are aberrantly expressed in a number of cancers, including EOC where they regulate tumour growth (7). Circulating miRs are stable in the blood and reflect changes in the distant tumour allowing a 'liquid biopsy' of the tumour to be non-invasively obtained.

In the present study, we examined the expression of five miRs that are involved in the pathogenesis of EOC, although, to the best of our knowledge, their expression in blood has 
not been investigated or consistently reproduced in previous studies. We found significant upregulation of the miR-200 family in EOC compared to benign ovarian tumours. The miR-200 family likely plays a key role in carcinogenesis. They are critical regulators of the epithelial-mesenchymal transition (7). This process describes the transformation of non-motile epithelial to motile mesenchymal cells, which therefore have the potential to invade surrounding tissue and metastasize to distant sites. The miR-200s target two E-box binding transcription factors, ZEB1 and ZEB2, which regulate E-cadherin expression and cellular polarity (7). Increased ovarian tissue expression of miR-200a and miR-200c have been associated with a reduced overall survival in EOC patients, which may be attributed to alterations in response to chemotherapeutics with high levels of miR-200s $(8,9)$. As a result, the miR-200 family may not only be used to detect and stage EOC but may provide information regarding prognosis.

miR-210 has been shown to be increased in EOC tissue and cell lines in response to tissue hypoxia; however, little is known with regard to circulating miR-210 in EOC (10). miR-21 has been identified as an important oncogene in multiple types of cancer and has been shown to be increased in EOC tissue; nevertheless, little is known regarding circulating miR-21 in EOC $(5,11)$. In this pilot study of a limited number of patients, we saw a trend towards increased miR-210 with advanced EOC, but which did not meet statistical significance. The potential of miR-210 and miR-21 as useful EOC biomarkers requires further investigation in a larger cohort study.

Circulating miRs may be detected in whole blood, plasma or serum. To the best of our knowledge, this is the first study to describe their expression in EOC in whole blood collected prior to initial surgery (12). Whole blood collected directly into tubes containing RNA stabilizer (PAXgene tubes) ensures that the cells are lysed and RNA stabilized immediately to inhibit ex vivo transcription. By contrast, the expression of miRs in plasma and serum have consistently been shown to be affected by ex vivo sample handling (13). This may account for the differing results obtained in studies with different collection methods. Whole blood overcomes these differences, and may be an ideal medium for sample collection in large multi-centre studies.

A strength of this study is that it was prospective in design and we only included high-grade serous EOC patients. Many biomarker studies include serous, endometriod, clear cell and non-EOC altogether. Although it would be ideal to develop a biomarker that can detect all forms of ovarian cancer, there is a difference in the pathophysiology and genomics of each histological subtype and therefore EOC should be considered separately in biomarker discovery experiments (14). Nevertheless, although our results are promising, there are important limitations. These include the small sample size in this pilot study, which restricts the ability to determine the accuracy of these miRs to diagnose EOC. Therefore, these preliminary findings require further validation to assess the diagnostic potential of circulating miRs in ovarian cancer.
In conclusion, circulating miRs, specifically from the miR-200 family, are increased in high-grade serous EOC, reflect the extent of disease and constitute potential candidate biomarkers for non-invasive screening for EOC. Large validation studies are required to develop biomarkers to ensure early diagnosis and timely treatment to improve outcomes for women with EOC.

\section{Acknowledgements}

We would like to thank Gabrielle Fleming, research nurse for assisting in subject recruitment and sampling.

\section{References}

1. Siegel R, Naishadham D and Jemal A: Cancer statistics, 2012. CA Cancer J Clin 62: 10-29, 2012.

2. Scholz HS, Tasdemir H, Hunlich T, Turnwald W, Both A and Egger H: Multivisceral cytoreductive surgery in FIGO stages IIIC and IV epithelial ovarian cancer: Results and 5-year follow-up. Gynecol Oncol 106: 591-595, 2007.

3. Buys SS, Partridge E, Black A, Johnson CC, Lamerato L, Isaacs C, Reding DJ, Greenlee RT, Yokochi LA, Kessel B, et al; PLCO Project Team: Effect of screening on ovarian cancer mortality: The Prostate, Lung, Colorectal and Ovarian (PLCO) Cancer Screening Randomized Controlled Trial. JAMA 305: 2295-2303, 2011.

4. Shen J, Stass SA and Jiang F: MicroRNAs as potential biomarkers in human solid tumors. Cancer Lett 329: 125-136, 2013.

5. Pal MK, Jaiswar SP, Dwivedi VN, Tripathi AK, Dwivedi A and Sankhwar P: MicroRNA: A new and promising potential biomarker for diagnosis and prognosis of ovarian cancer. Cancer Biol Med 12: 328-341, 2015.

6. Harris AL: Hypoxia - a key regulatory factor in tumour growth. Nat Rev Cancer 2: 38-47, 2002.

7. Bendoraite A, Knouf EC, Garg KS, Parkin RK, Kroh EM, O'Briant KC, Ventura AP, Godwin AK, Karlan BY, Drescher CW, et al: Regulation of miR-200 family microRNAs and ZEB transcription factors in ovarian cancer: Evidence supporting a mesothelial-to-epithelial transition. Gynecol Oncol 116: 117-125, 2010

8. Kim SW, Kim JW, Kim YT, Kim JH, Kim S, Yoon BS, Nam EJ and Kim HY: Analysis of chromosomal changes in serous ovarian carcinoma using high-resolution array comparative genomic hybridization: Potential predictive markers of chemoresistant disease. Genes Chromosomes Cancer 46: 1-9, 2007.

9. Zuberi M, Mir R, Das J, Ahmad I, Javid J, Yadav P, Masroor M, Ahmad S, Ray PC and Saxena A: Expression of serum miR-200a, miR-200b, and miR-200c as candidate biomarkers in epithelial ovarian cancer and their association with clinicopathological features. Clin Transl Oncol 17: 779-787, 2015.

10. Li L, Huang K, You Y, Fu X, Hu L, Song L and Meng Y: Hypoxia-induced miR-210 in epithelial ovarian cancer enhances cancer cell viability via promoting proliferation and inhibiting apoptosis. Int J Oncol 44: 2111-2120, 2014.

11. Chen Y, Chen Q, Liu Q and Gao F: Human epididymis protein 4 expression positively correlated with miR-21 and served as a prognostic indicator in ovarian cancer. Tumour Biol 37: 8359-8365, 2016.

12. Häusler SF, Keller A, Chandran PA, Ziegler K, Zipp K, Heuer S, Krockenberger M, Engel JB, Hönig A, Scheffler M, et al: Whole blood-derived miRNA profiles as potential new tools for ovarian cancer screening. Br J Cancer 103: 693-700, 2010.

13. Malentacchi F, Pizzamiglio S, Wyrich R, Verderio P, Ciniselli C, Pazzagli M and Gelmini S: Effects of Transport and Storage Conditions on Gene Expression in Blood Samples. Biopreserv Biobank 14: 122-128, 2016.

14. Bell D, Berchuck A, Birrer M, Chien J, Cramer DW, Dao F, Dhir R, DiSaia P, Gabra H, Glenn P, et al; Cancer genome atlas research network: Integrated genomic analyses of ovarian carcinoma. Nature 474: 609-615, 2011. 